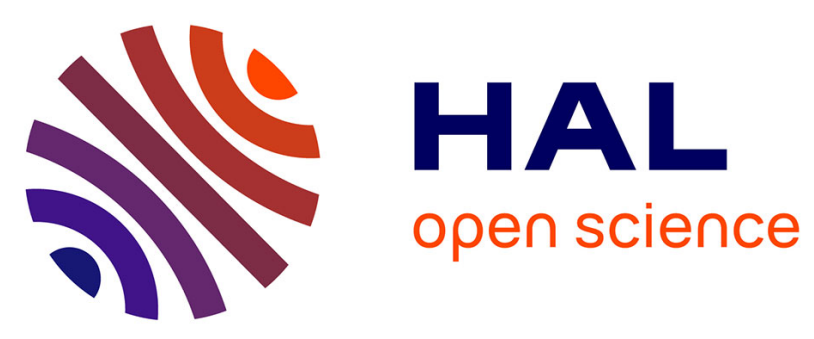

\title{
Chebyshev Knots
}

Pierre-Vincent Koseleff, Daniel Pecker

\section{To cite this version:}

Pierre-Vincent Koseleff, Daniel Pecker. Chebyshev Knots. Journal of Knot Theory and Its Ramifications, 2011, 20 (4), pp.575-593. 10.1142/S0218216511009364 . hal-00344501v2

\section{HAL Id: hal-00344501 \\ https://hal.science/hal-00344501v2}

Submitted on 8 Apr 2010

HAL is a multi-disciplinary open access archive for the deposit and dissemination of scientific research documents, whether they are published or not. The documents may come from teaching and research institutions in France or abroad, or from public or private research centers.
L'archive ouverte pluridisciplinaire HAL, est destinée au dépôt et à la diffusion de documents scientifiques de niveau recherche, publiés ou non, émanant des établissements d'enseignement et de recherche français ou étrangers, des laboratoires publics ou privés. 


\title{
Chebyshev knots
}

\author{
P. -V. Koseleff*, D. Pecker ${ }^{\dagger}$ \\ Université Pierre et Marie Curie \\ 4, place Jussieu, F-75252 Paris Cedex 05 \\ e-mail: $\{$ koseleff,pecker\}@math.jussieu.fr
}

April 8, 2010

\begin{abstract}
A Chebyshev knot is a knot which admits a parametrization of the form $x(t)=$ $T_{a}(t) ; y(t)=T_{b}(t) ; z(t)=T_{c}(t+\varphi)$, where $a, b, c$ integers, $T_{n}(t)$ is the Chebyshev polynomial of degree $n$, and $\varphi \in \mathbf{R}$. Chebyshev knots are non compact analogues of the classical Lissajous knots. We show that there are infinitely many Chebyshev knots with $\varphi=0$. We also show that every knot is a Chebyshev knot.

keywords: Polynomial curves, Chebyshev polynomials, Chebyshev curves, Lissajous knots, long knots, braids

Mathematics Subject Classification 2000: 14H50, 57M25, 14P99
\end{abstract}

\section{Introduction}

A Lissajous knot is a knot which admits a one-to-one parametrization of the form

$$
x=\cos (a t) ; y=\cos (b t+\varphi) ; z=\cos (c t+\psi)
$$

where $0 \leq t \leq 2 \pi$ and where $a, b, c$ are pairwise coprime integers. These knots, first defined in [2], have been studied by many authors: V. F. R. Jones, J. Przytycki, C. Lamm, J. Hoste and L. Zirbel. Most known properties of Lissajous knots are deduced from their symmetries, which are easy to see (see [9, 16, 8, 20]).

On the other hand Vassiliev considered polynomial knots, i.e. non singular polynomial embeddings $\mathbf{R} \rightarrow \mathbf{R}^{3}$ (see [25, 23, 21, 11, 12]).

In this paper we study a polynomial analogue of Lissajous knots. It is natural to use the classical Chebyshev polynomials $T_{n}(t)$ instead of cosine functions to define our Chebyshev knots. The Chebyshev polynomials are defined by $T_{0}=1, T_{1}=t, T_{n+1}=2 t T_{n}-T_{n-1}, n \in$ Z. They satisfy the trigonometric identity $\cos (n \theta)=T_{n}(\cos \theta)$.

*Faculté de Mathématiques and Salsa-Inria project

${ }^{\dagger}$ Faculté de Mathématiques 
Definition 1 A knot in $\mathbf{R}^{3} \subset \mathbf{S}^{3}$ is a Chebyshev knot if it admits a one to one parametrization of the form

$$
x=T_{a}(t) ; y=T_{b}(t) ; z=T_{c}(t+\varphi)
$$

where $t \in \mathbf{R}, a, b, c$ are integers and $\varphi$ is a real constant.

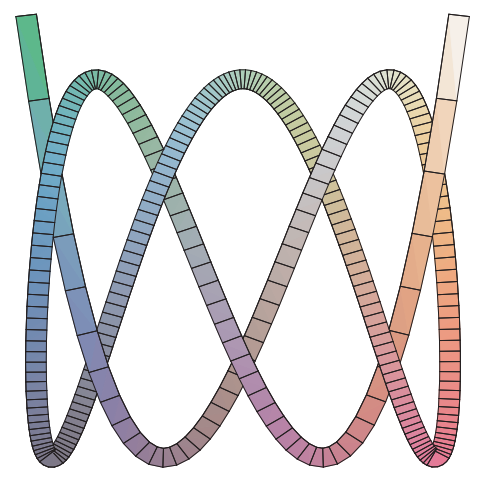

Figure 1: The mirror image of the $7_{7}$ knot is Chebyshev.

We begin with the study of plane Chebyshev curves which are projections of Chebyshev knots on the $(x, y)$-plane. We conclude this paragraph with a theorem of Hoste and Zirbel [8] describing these curves in terms of particular braid projections.

Then, we study some families of Chebyshev knots with $\varphi=0$, called harmonic knots. We prove that for $a, b$ coprime positive integers and $c=a b-a-b$, the harmonic knot $\mathrm{H}(a, b, c)$ has an alternating projection on the $(x, y)$-plane. We deduce that there are infinitely many harmonic knot types. This is similar to a theorem of C. Lamm concerning Lissajous knots (see [16]). We also prove that the torus knots $\mathrm{T}(2,2 n+1)$ are harmonic knots. On the other hand, we observe that the symmetries of harmonic knots are quite different from those of Lissajous knots. There are infinitely many amphicheiral harmonic knots and infinitely many strongly reversible harmonic knots. The trefoil and the figure-eight knot are harmonic knots but are not Lissajous. Some knots are both Lissajous and harmonic knots, e.g. $5_{2}$ and $7_{5}$.

We conclude the paper with our principal result: every knot is a Chebyshev knot. This is done by showing first that every knot has a plane projection which is a Chebyshev curve. Then we use some classical results of braid theory and a density argument based on Kronecker's theorem.

At the end we give Chebyshev diagrams of the first 2-bridge harmonic knots.

\section{Geometry of plane Chebyshev curves}

Chebyshev curves were defined in [5] to replace the older denomination of "doubly parametrized Lissajous curves". Their double points are easier to study than those of Lissajous 
curves. It will be convenient to consider also the case of implicit Chebyshev curves.

Proposition 1 Let $a, b$ be nonnegative integers, a being odd. The affine Chebyshev curve $\mathcal{C}$ defined by

$$
\mathcal{C}: \quad T_{b}(x)-T_{a}(y)=0
$$

has $\frac{1}{2}(a-1)(b-1)$ singular points which are crossing points. These points form two rectangular grids contained in the open square $Q=(-1,1)^{2}, R=\left\{(x, y) \in Q, T_{b}(x)=T_{a}(y)=1\right\}$, and $R^{\prime}=\left\{(x, y) \in Q, T_{b}(x)=T_{a}(y)=-1\right\}$.

Proof. The singular points of $\mathcal{C}$ are obtained for $T_{b}^{\prime}(x)=0, T_{a}^{\prime}(y)=0, T_{b}(x)=T_{a}(y)$. From $T_{a}(\cos \theta)=\cos a \theta$, we deduce that $T_{a}$ has degree $a$ and $T_{a}^{\prime}(\cos \theta)=a \frac{\sin a \theta}{\sin \theta} . T_{a}^{\prime}$ has $a-1$ simple roots in $(0,1): y_{k}=\cos \left(k \frac{\pi}{a}\right), k=1, \ldots, a-1$. At these points, we have $T_{a}\left(y_{k}\right)=(-1)^{k}$. $T_{b}^{\prime}$ has $b-1$ roots in $(0,1): x_{1}, \ldots, x_{b-1}$. For each $x_{i}$ there are exactly $\frac{1}{2}(a-1)$ values $y_{j}$ satisfying $T_{a}^{\prime}\left(y_{j}\right)=0, T_{a}\left(y_{j}\right)=T_{b}\left(x_{i}\right)$. Hence the number of singular points is $\frac{1}{2}(a-1)(b-1)$, and they form two rectangular grids. Since the roots of $T_{b}^{\prime}(x)=0$ are simple, we see that these points are crossing points.

Remark 1 It follows from their definitions that $|R|=\frac{1}{2}\left[\frac{b-1}{2}\right](a-1),\left|R^{\prime}\right|=\frac{1}{2}\left[\frac{b}{2}\right](a-1)$ where $\lfloor x\rfloor$ is the greatest integer less than or equal to $x$.

Proposition 2 Let $a$ and $b$ are nonnegative coprime integers, a being odd. Let the Chebyshev curve $\mathcal{C}$ be defined by the equation $T_{b}(x)-T_{a}(y)=0$. Then $\mathcal{C}$ admits the parametrization $x=T_{a}(t), y=T_{b}(t)$. The pairs $(t, s)$ giving a crossing point are

$$
t=\cos \left(\frac{k}{a}+\frac{h}{b}\right) \pi, s=\cos \left(\frac{k}{a}-\frac{h}{b}\right) \pi
$$

where $k, h$ are positive integers such that $\frac{k}{a}+\frac{h}{b}<1$.

Proof. Since $T_{a} \circ T_{b}=T_{b} \circ T_{a}=T_{a b}$, the rational curve $\mathcal{C}^{\prime}$ parametrized by $x=T_{a}(t), y=$ $T_{b}(t)$ is contained in $\mathcal{C}$. These two curves intersect the line $\left\{x=x_{0}\right\}$ in one point if $\left|x_{0}\right|>1$, in $a$ points if $\left|x_{0}\right|<1$ and in $\frac{1}{2}(a+1)$ points if $x_{0}= \pm 1$. Consequently, they are equal.

The $\frac{1}{2}(a-1)(b-1)$ pairs

$$
t=\cos \left(\frac{k}{a}+\frac{h}{b}\right) \pi, s=\cos \left(\frac{k}{a}-\frac{h}{b}\right) \pi,
$$

give rise to double points of $\mathcal{C}^{\prime}=\mathcal{C}$. Because the number of singular points of $\mathcal{C}$ is $\frac{1}{2}(a-$ $1)(b-1)$, we see that there is no other singular point. 
Remark 2 We observe that the crossing points are obtained for the $(a-1)(b-1)$ elements of

$$
E=\left\{t_{u}=\cos \frac{u}{a b} \pi, 0 \leq u \leq a b, a \nmid u, b \nmid u\right\} .
$$

For these values, we get $T_{b}\left(x\left(t_{u}\right)\right)=T_{a}\left(y\left(t_{u}\right)\right)=(-1)^{u}$. Note that $t_{u}$ and $t_{u^{\prime}}$ correspond to the same point when $u \equiv-u^{\prime}(\bmod 2 b)$ and $u \equiv u^{\prime}(\bmod 2 a)$.

Remark 3 In general, the curve $\mathcal{C}: T_{b}(x)-T_{a}(y)=0$ has $\left[\frac{d}{2}\right]+1$ components where $d=\operatorname{gcd}(a, b)$. See Figure 团.

The following proposition will be useful to consider Chebyshev curves as trajectories in a rectangular billiard (see [9]).

Proposition 3 Let $\mathcal{C}$ be the Chebyshev curve: $T_{b}(x)-T_{a}(y)=0$. There exists an homeomorphism from the square $I^{2}=[-1,1]^{2}$ to the rectangle $[0, b] \times[0, a]$, such that the image of $\mathcal{C} \cap I^{2}$ is the union of all the billiard trajectories with slopes \pm 1 through the points with coordinates $x=b, y=a-2 k, 0 \leq 2 k \leq a$.

Proof. Consider the mapping $F(x, y)=(X, Y)$ with $\pi X=b(\pi-\arccos x), \pi Y=a(\pi-$ $\arccos y)$. By trigonometry, it is not hard to check that $F$ has the announced properties.
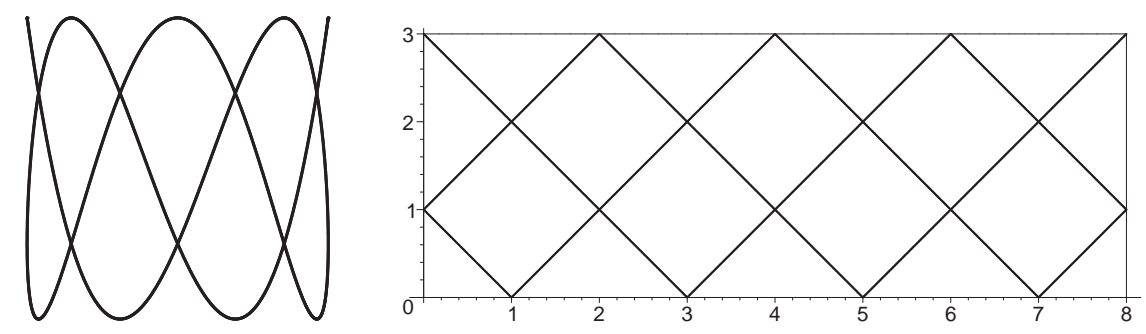

Figure 2: $T_{8}(x)=T_{3}(y)$ and its billiard picture

We shall now present a description of Chebyshev curves using braids. Let $B_{n}$ be the group of braids on $n$ strings. For practical purposes we shall draw these braids horizontally, the strings being numbered from the bottom to the top. The standard braid generators are denoted $\sigma_{1}, \sigma_{2}, \ldots, \sigma_{n-1}$. The braid $\sigma_{i}$ exchanges the strings $i+1$ and $i$, the string $i+1$ passing over the string $i$. In this paragraph we shall be interested in plane projections of braids, called plane braids. We shall also consider the composition of such plane braids. Let $a=n$. Let $s_{i}$ denote the plane braid which is the plane projection of $\sigma_{i}$. This plane braid has one crossing point.

Following Hoste and Zirbel [ [8], let us define the plane braids $s_{\text {even }}$ and $s_{\text {odd }}$ as

$$
s_{\text {even }}=s_{2} s_{4} \cdots s_{E}, \quad s_{\text {odd }}=s_{1} s_{3} \cdots s_{O},
$$

where $E$ and $O$ are the largest even and odd integers less than $n=a$. 

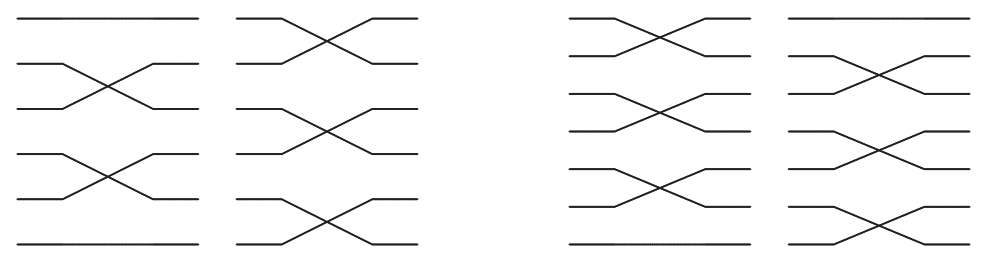

Figure 3: $s_{\text {even }}$ and $s_{\text {odd }}$ for $n=6,7$.

Proposition 4 Let $a, b$ be integers, a being odd. Let $\mathcal{C}$ be the Chebyshev curve $T_{b}(x)-$ $T_{a}(y)=0$. Let $\varepsilon>0$ small enough and consider the rectangle $R_{\varepsilon}=\{|x|<1-\epsilon,|y| \leq$ $1\}$. Then there is a homeomorphism between the pairs $\left(R_{\varepsilon}, \mathcal{C}\right)$ and $\left(R_{\varepsilon}, \rho\right)$ where $\rho=$ $\left(s_{\text {odd }} s_{\text {even }}\right)^{\frac{b-1}{2}}$ if $b$ is odd and $\rho=\left(s_{\text {even }} s_{\text {odd }}\right)^{\frac{b-2}{2}} s_{\text {even }}$ if $b$ is even.

Proof. Following the proof of Proposition 1, the $\frac{1}{2}(a-1)(b-1)$ singular points of $\mathcal{C}$ are in $R_{\varepsilon}$ when $\varepsilon$ is small enough. For each $k=1, \ldots, b-1$, there are $\frac{1}{2}(a-1)$ singular points

$$
\left(x_{k}, y_{l}\right)=\left(\cos k \frac{\pi}{b}, \cos l \frac{\pi}{a}\right), k+l \equiv 0(\bmod 2) .
$$

It means that over a neighborhood over $x_{k}$, the curve $\mathcal{C}$ is isotopic to $s_{\text {even }}$ if $k$ is odd, and isotopic to $s_{\text {odd }}$ if $k$ is even. This proves the result.

We can define the plat closure of a plane horizontal braid with $2 m$ strings labelled $0,1, \ldots, 2 m-$ 1 , to be the plane curve obtained by connecting the right ends 0 to $1, \ldots, 2 m-2$ to $2 m-1$, and the left ends in the same order.

Corollary 1 Let a be an odd integer, and $b$ an even integer. Let $\rho^{\prime}$ be the plane braid with $a+1$ strings obtained by adding a free string numbered $a+1$ over $\rho=\left(s_{\text {even }} s_{\text {odd }}\right)^{\frac{b-2}{2}} s_{\text {even }}$. Then the Chebyshev curve $T_{b}(x)-T_{a}(y)=0$ is isotopic (in $\left.\mathbf{S}^{2}\right)$ to the plat closure of the plane braid $\rho^{\prime}$.

Proof. Let us illustrate this by looking at the curve $T_{10}(x)-T_{5}(y)=0$, which has 3 components. We see on Figure 4 that it is the plat closure of $\left(s_{\text {even }} s_{\text {odd }}\right)^{4} s_{\text {even }}$.

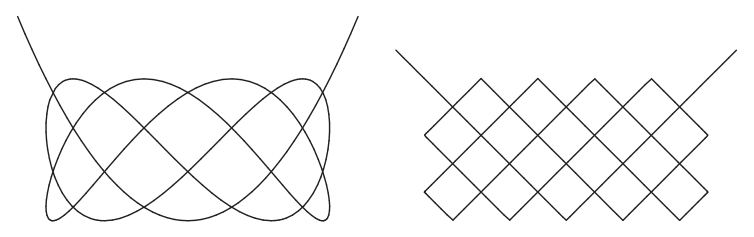

Figure 4: The Chebyshev curve $T_{10}(x)-T_{5}(y)=0$ and its billiard picture 


\section{Harmonic knots}

In this paragraph we shall study Chebyshev knots with $\varphi=0$. Comstock (1897) found the number of crossing points of the harmonic curve parametrized by $x=T_{a}(t), y=T_{b}(t), z=$ $T_{c}(t)$. In particular, he proved that this curve is non singular if and only if $a, b, c$ are pairwise coprime integers [四. Such curves will be named harmonic knots $\mathrm{H}(a, b, c)$.

We see that $\mathrm{H}(a, b, 1)$ is the unknot because the height function is monotonic. We can also obtain the unknot in a less trivial way.

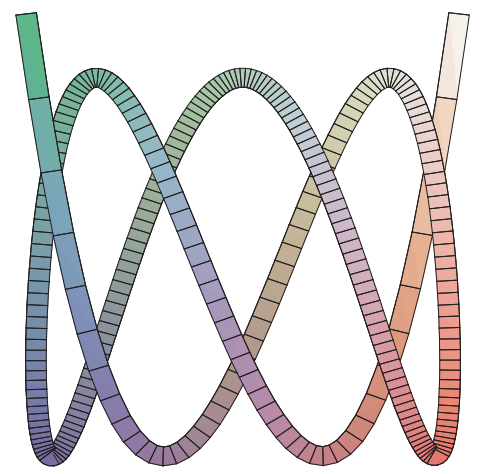

Figure 5: The knot $\mathrm{H}(3,8,11)$ is trivial.

Proposition 5 Let $a, b$ be coprime integers and $c=a+b$. The harmonic knot $\mathrm{H}(a, b, c)$ is trivial.

Proof. Let $t \in[-1,1], t=\cos \theta$. We have $x=T_{a}(t)=\cos a \theta, y=\cos b \theta, z=\cos (a+b) \theta$. By trigonometry, we see that the bounded part of our knot is on the surface

$$
S=\left\{(x, y, z) \in \mathbf{R}^{3},|x| \leq 1,|y| \leq 1, z=x y \pm \sqrt{\left(1-x^{2}\right)\left(1-y^{2}\right)}\right\} .
$$

Since $S$ is the union of two sheets that are homeomorphic to the square $[0,1]^{2}$ glued along their boundaries, we see that it is homeomorphic to a sphere. Consequently the genus of $\mathrm{H}(a, b, c)$ is zero, hence it is the unknot.

Note that the surface $S$ has the symmetries of a regular tetrahedron. It is contained in the cubic surface $\left\{x^{2}+y^{2}+z^{2}=1+2 x y z\right\}$ which has the same symmetries.

Let $\mathcal{C}$ be a plane projection of a parametrized knot. Consider a crossing point of $\mathcal{C}$ obtained for the parameter pair $(t, s)$. The tangents at this point have opposite slopes (see 15], Lemma 4). It follows easily that the nature of this crossing point depends only of the sign of the expression $D=(z(t)-z(s)) x^{\prime}(t) y^{\prime}(t)$. This is not the usual definition of the sign of oriented crossings, see Figure 6 . 


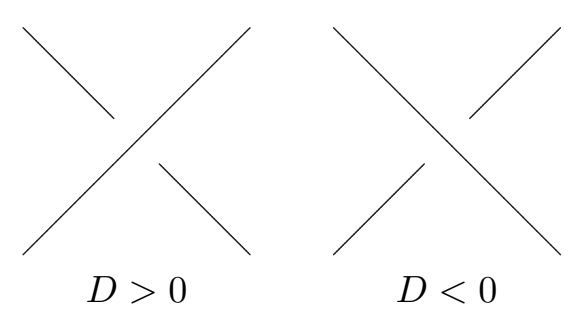

Figure 6: The right twist and the left twist

Lemma 1 Let $\mathrm{H}(a, b, c)$ be a harmonic knot. The nature of the crossing point of parameter $t=\cos \left(\frac{k}{a}+\frac{h}{b}\right) \pi$, is given by

$$
\operatorname{sign}(D)=\operatorname{sign}\left(-(-1)^{h+k} \sin \left(\frac{a h}{b} \pi\right) \sin \left(\frac{b k}{a} \pi\right) \sin \left(\frac{c h}{b} \pi\right) \sin \left(\frac{c k}{a} \pi\right)\right) .
$$

Proof. The crossing points of the plane projection $\left\{x=T_{a}(t), y=T_{b}(t)\right\}$ are obtained for the parameters $t=\cos \tau, s=\cos \sigma$, where

$$
\tau=\left(\frac{k}{a}+\frac{h}{b}\right) \pi, \sigma=\left(\frac{k}{a}-\frac{h}{b}\right) \pi .
$$

Using trigonometry we get $x^{\prime}(t)=a \frac{\sin a \tau}{\sin \tau}, y^{\prime}(t)=b \frac{\sin b \tau}{\sin \tau}$, so

$$
x^{\prime}(t) y^{\prime}(t)=(-1)^{h+k} \frac{a b}{\sin ^{2} \tau} \sin \left(\frac{a h}{b} \pi\right) \sin \left(\frac{b k}{a} \pi\right) .
$$

We have also

$$
z(t)-z(s)=T_{c}(t)-T_{c}(s)=-2 \sin \left(\frac{c h}{b} \pi\right) \sin \left(\frac{c k}{a} \pi\right) .
$$

and the announced result.

\section{Alternate harmonic knots}

The following theorem is the analogue of a theorem of Lamm [16] concerning Lissajous knots.

Theorem 1 (Alternate harmonic knots) Let $a, b$ be positive coprime integers, and $c=$ $a b-a-b$. The harmonic diagram $\mathrm{H}(a, b, c)$ is alternating.

Proof. Using Equation (3), we get

$$
\begin{aligned}
z(t)-z(s) & =-2 \sin \left(\frac{c h}{b} \pi\right) \sin \left(\frac{c k}{a} \pi\right) \\
& =-2 \sin \left((a-1) h \pi-\frac{a h}{b} \pi\right) \sin \left((b-1) k \pi-\frac{b k}{a} \pi\right) \\
& =-2(-1)^{h(a-1)+k(b-1)} \sin \left(\frac{a h}{b} \pi\right) \sin \left(\frac{b k}{a} \pi\right) .
\end{aligned}
$$


Using Equation (2), we get $\operatorname{sign}(D)=-(-1)^{a h+b k}$. The crossing points are obtained for the $(a-1)(b-1)$ elements of $E=\left\{t_{u}=\cos \frac{u}{a b} \pi, 0 \leq u \leq a b, a \nmid u, b \backslash u\right\}$.

Note that $t_{u}<t_{u-1}$ and that at the crossing point corresponding to $t_{u} \in E$ one has $\operatorname{sign}(D)=-(-1)^{u}$.

The polynomial $x^{\prime}(t) y^{\prime}(t)$ has $(a+b-2)$ simple roots for $t_{u}=\cos \frac{u}{a b} \pi$, where $a$ or $b$ divides $u=1, \ldots, a b-1$. For these parameters, the billiard curve corresponding to the $(x, y)$-plane projection bounces on a wall.

Three cases may occur because at least one of three consecutive $t_{u}$ belongs to $E$.

1. $t_{h+1} \in E$ and $t_{h} \in E$. Then sign $\left(x^{\prime}\left(t_{h}\right) y^{\prime}\left(t_{h}\right)\right)=\operatorname{sign}\left(x^{\prime}\left(t_{h+1}\right) y^{\prime}\left(t_{h+1}\right)\right)$ and since the sign of $D$ changes, we conclude that the sign of $z(t)-z(s)$ changes between the 2 consecutive parameters $t_{h+1}$ and $t_{h}$.

2. $t_{h+1} \in E, t_{h} \notin E, t_{h-1} \notin E$. We have $x^{\prime}(t) y^{\prime}(t)=0$ at $t_{h}$ and $t_{h-1}$. For $t_{h-1}<t<$ $t_{h-2}$, we have sign $\left(x^{\prime}(t) y^{\prime}(t)\right)=\operatorname{sign}\left(x^{\prime}\left(t_{h+1}\right) y^{\prime}\left(t_{h+1}\right)\right)$, so $\operatorname{sign}\left(x^{\prime}\left(t_{h-2}\right) y^{\prime}\left(t_{h-2}\right)\right)=$ $\operatorname{sign}\left(x^{\prime}\left(t_{h+1}\right) y^{\prime}\left(t_{h+1}\right)\right)$. Hence we see that the sign of $z(t)-z(s)$ changes between the 2 consecutive parameters $t_{h+1}$ and $t_{h-2}$.

3. $t_{h+1} \in E, t_{h} \notin E, t_{h-1} \in E$. We have $x^{\prime}(t) y^{\prime}(t)=0$ at $t_{h}$, so $\operatorname{sign}\left(x^{\prime}\left(t_{h-1}\right) y^{\prime}\left(t_{h-1}\right)\right)=$ $-\operatorname{sign}\left(x^{\prime}\left(t_{h+1}\right) y^{\prime}\left(t_{h+1}\right)\right)$. Hence we see that the sign of $z(t)-z(s)$ changes between the 2 consecutive parameters $t_{h+1}$ and $t_{h-1}$.

In conclusion, the diagram is alternating.

\section{Symmetries and harmonic knots}

A knot $K$ in $\mathbf{S}^{3}$ is strongly (-)amphicheiral if there is an involution of $\left(\mathbf{S}^{3}, K\right)$ which reverses the orientation of both $\mathbf{S}^{3}$ and $K$. A knot $K$ in $\mathbf{S}^{3}$ is strongly reversible (or strongly invertible) if there is an involution of $\left(\mathbf{S}^{3}, K\right)$ which preserves the orientation of $\mathbf{S}^{3}$ and reverses the orientation of $K$ (see [10], pp. 127-128).

Proposition 6 The harmonic knot $\mathrm{H}(a, b, c)$ is either strongly (-)amphicheiral if abc is odd, or strongly reversible if abc is even.

Proof. It is immediate from the parity of Chebyshev polynomials.

Corollary 2 There are infinitely many amphicheiral harmonic knots. There are infinitely many strongly reversible harmonic knots.

Proof. Since the harmonic knot $\mathrm{H}(a, b, c), c=a b-a-b$ is alternate, its crossing number is $\frac{1}{2}(a-1)(b-1)$. From this we conclude that there is an infinity of such knots with $a, b, c$ odd, or with $a b c$ even. 
If $\sigma$ is any permutation of $\{a, b, c\}$ then the harmonic knot $\mathrm{H}(\sigma(a), \sigma(b), \sigma(c))$ is either $\mathrm{H}(a, b, c)$ if $\sigma$ is an even permutation or its mirror image if $\sigma$ is an odd permutation.

Proposition 7 Let $a, b$ be coprime integers. $\mathrm{H}(a, b, c), \mathrm{H}(a, b, 2 a b-c)$ and $\mathrm{H}(a, b, 2 a b+c)$ are the same knot.

Proof. The expression of sign $(D)$ (Equations (2) and (3)) for a given pair of parameters $(t, s)$ corresponding to crossing points in the $(x, y)$-plane projection is invariant under the transformation $c \mapsto c+2 a b$ and $c \mapsto 2 a b-c$.

We can therefore suppose that $a<b$ and $0<c<a b$ to consider all cases.

Proposition 8 Let $a, b, c$ be relatively prime integers. There exists $c^{\prime}$ such that $\mathrm{H}\left(a, b, c^{\prime}\right)$ is the mirror image of $\mathrm{H}(a, b, c)$.

Proof. Because $a$ and $b$ are relatively prime, one can write $c=\alpha a+\beta b$ where $\alpha$ and $\beta$ are integers. Let us consider $c^{\prime}=-\alpha a+\beta b$. We have $c^{\prime} \equiv c(\bmod 2 a)$ and $c^{\prime} \equiv-c(\bmod 2 b)$. For any crossing point of the Chebyshev curve $\mathcal{C}: T_{b}(x)=T_{a}(y)$ corresponding to diagrams of both $\mathrm{H}(a, b, c)$ and $\mathrm{H}\left(a, b, c^{\prime}\right)$, we see that $\operatorname{sign}(D)$ changes to opposite when $c$ is replaced by $c^{\prime}$.

Corollary $3 \mathrm{H}(a, b, a b+a-b)$ is the mirror image of the alternate knot $\mathrm{H}(a, b, a b-a-b)$.

Corollary 4 Let $a, b$ be relatively prime integers. There are at most $\varphi(a) \varphi(b)$ different harmonic knots $\mathrm{H}(a, b, c)$.

Proof. The number of $c$ in $[1, a b]$ that are relatively prime to $a$ and $b$ is $\varphi(a) \varphi(b)$ where $\varphi$ is the Euler function.

Remark 4 Because of Propositions 0 and 8, for each $c$ there exists $c^{\prime}<a b$ such that $\mathrm{H}\left(a, b, c^{\prime}\right)$ is the mirror image of $\mathrm{H}(a, b, c)$. We have at most $\frac{1}{2} \varphi(a) \varphi(b)$ different knots when we identify the knots and their mirror images.

When $a+1<b, \mathrm{H}(a, b, 1)$ and its mirror image, $\mathrm{H}(a, b, a+b)$ and $\mathrm{H}(a, b, b-a)$ are trivial.

Independently but later, G. and J. Freudenburg (国) proved the following improvement of Proposition 8: There is a polynomial automorphism $\Phi$ of $\mathbf{R}^{3}$ such that $\Phi(\mathrm{H}(a, b, c))=$ $\mathrm{H}\left(a, c^{\prime}, b\right)$. They deduce another proof that the harmonic knots $\mathrm{H}(a, b, a+b)$ are trivial. 

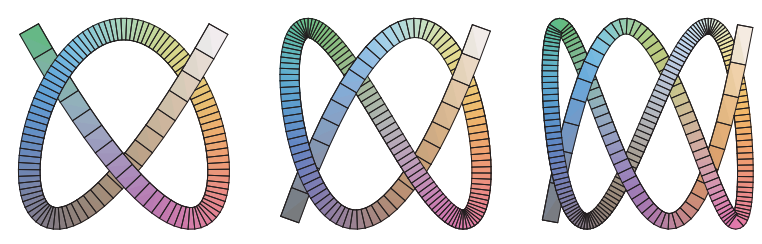

Figure 7: The trefoil, the figure-eight knot, and the $6_{3}$ knot.

\section{The simplest alternate harmonic knots}

It is remarkable that for $a=3$ the curves are drawn in "Conway normal form" for 2-bridge knots [18]. Then their Conway notation is $\mathrm{H}(3, n, 2 n-3)=C(1,1, \ldots, 1)$ when $n$ is not a multiple of 3. Turner [24] named these knots Fibonacci knots, because their determinants are Fibonacci numbers. For $n=4$ we obtain the trefoil, for $n=5$ the figure-eight, for $n=7$ the $6_{3}$ knot, and for $n=8$ the $\overline{7}_{7}$ knot.

The Fibonacci knots with an even crossing number are 2-bridge amphicheiral knots. We have recently proved ([14]) that they are not Lissajous.

For $a=4$, we also obtain 2-bridge knots. Following the classical method ([18] p. 183187), we see that their Conway notation is $\mathrm{H}(4, n, 3 n-4)=C(-1,-2, \ldots,-1,-2)=$ $C(-3,-1,-2, \ldots,-1,-2)$, $(n$ odd $)$. For $n=5$ we obtain the $6_{2}$ knot, for $n=7$ a symmetric
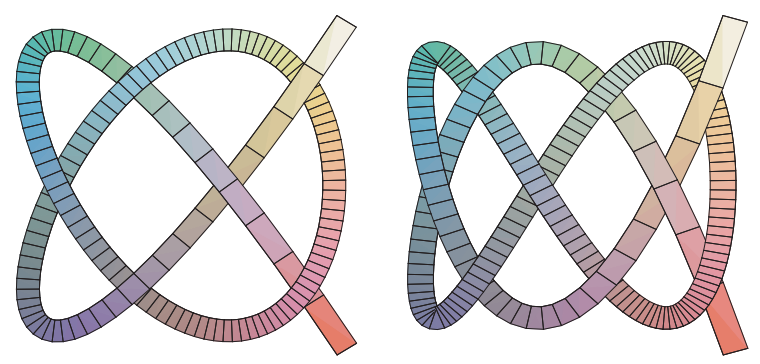

Figure 8: The $6_{2}$ and $9_{20}$ knots .

picture of the $9_{20}$ knot (compare with Rolfsen's table [22]).

For $a \geq 5$ we may obtain $p$-bridge knots, with $p \geq 3$. For example the harmonic knot $\mathrm{H}(5,6,19)$ is the mirror image of $10_{116}$ knot in Rolfsen's table (amazingly, with exactly the same picture). Its bridge number is known to be 3 .

Note that the torus knot $\mathrm{T}(2,2 n+1)$ cannot be obtained as an alternate harmonic knot, except for the trefoil knot, as it is proved in [11]. Nevertheless it can be obtained as an harmonic knot. 


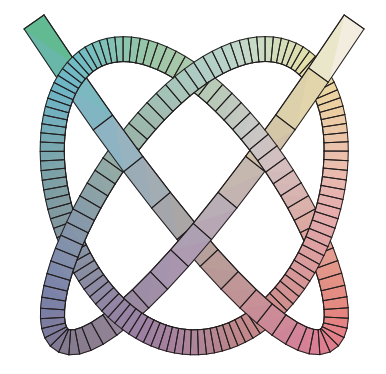

Figure 9: The mirror image of the $10_{116}$ knot.

The torus knots $\mathrm{T}(\mathbf{2}, \mathbf{2 n}+\mathbf{1})$

Theorem 2 The knot $\mathrm{H}(3,3 n+2,3 n+1)$ is the torus knot $\mathrm{T}(2,2 n+1)$.
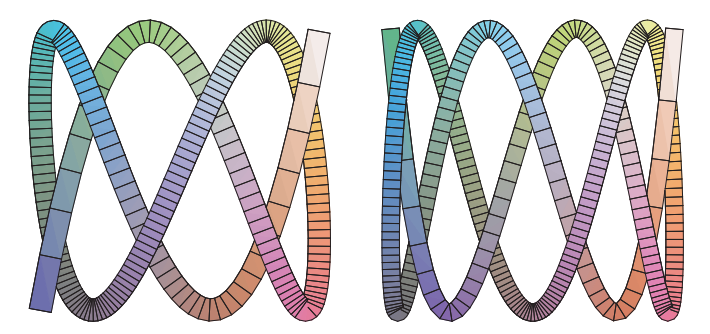

Figure 10: The torus knots $\overline{5}_{1}: \mathrm{H}(3,7,8)$ and $\overline{7_{1}}: \mathrm{H}(3,10,11)$

Proof. We shall determine the Conway normal form of the harmonic knot $\mathrm{H}(3, b, c), b=$ $3 n+1, c=b+1$. The crossing points of the plane projection of $\mathrm{H}(3, b, c)$ are obtained for pairs of values $(t, s)$ where $t=\cos \tau, s=\cos \sigma$, and $\tau=\frac{m}{3 b} \pi, \sigma=\frac{m^{\prime}}{3 b} \pi$.

For $k=0, \ldots, n-1$, let us consider

$A_{k}$ be obtained for $m=3 k+1, m^{\prime}=2 b-m$.

$B_{k}$ be obtained for $m=2 b+3 k+2, m^{\prime}=m-2 b$.

$C_{k}$ be obtained for $m=2 b+3 k+3, m^{\prime}=4 b-m$.

Then we have

$$
\begin{aligned}
& x\left(A_{k}\right)=\cos \left(\frac{3 k+1}{b} \pi\right), y\left(A_{k}\right)=\frac{1}{2}(-1)^{k} . \\
& x\left(B_{k}\right)=\cos \left(\frac{3 k+2}{b} \pi\right), y\left(B_{k}\right)=\frac{1}{2}(-1)^{k+1} .
\end{aligned}
$$




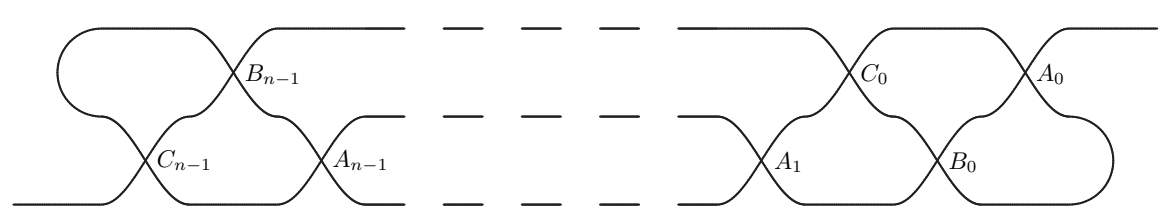

Figure 11: $\mathrm{H}(3,3 n+1, c), n$ even

$$
x\left(C_{k}\right)=\cos \left(\frac{3 k+3}{b} \pi\right), y\left(C_{k}\right)=\frac{1}{2}(-1)^{k} .
$$

Hence our $3 n$ points satisfy

$$
x\left(A_{k-1}\right)>x\left(B_{k-1}\right)>x\left(C_{k-1}\right)>x\left(A_{k}\right)>x\left(B_{k}\right)>x\left(C_{k}\right), k=1, \ldots, n-1 .
$$

Let us determine the nature of the crossing points. Using the identity $T_{a}^{\prime}(\cos \tau)=a \frac{\sin a \tau}{\sin \tau}$, we get

$$
\operatorname{sign}\left(x^{\prime}(t) y^{\prime}(t)\right)=\operatorname{sign}(\sin 3 \tau \sin b \tau) .
$$

We get

$$
\begin{aligned}
\text { for } A_{k}: \operatorname{sign}\left(x^{\prime}(t) y^{\prime}(t)\right) & =\operatorname{sign}\left(\sin \left(\frac{3 k+1}{b} \pi\right) \sin \left(\frac{3 k+1}{3} \pi\right)\right)=(-1)^{k} \\
\text { for } B_{k}: \operatorname{sign}\left(x^{\prime}(t) y^{\prime}(t)\right) & =\operatorname{sign}\left(\sin \left(\frac{2 b+3 k+2}{b} \pi\right) \sin \left(\frac{2 b+3 k+2}{3} \pi\right)\right) \\
& =(-1)^{k+1} \operatorname{sign}\left(\sin \left(\frac{3 k+2}{b} \pi\right) \sin \frac{\pi}{3}\right)=(-1)^{k+1} \\
\text { for } C_{k}: \operatorname{sign}\left(x^{\prime}(t) y^{\prime}(t)\right) & =\operatorname{sign}\left(\sin \left(\frac{2 b+3 k+3}{b} \pi\right) \sin \left(\frac{2 b+3 k+3}{3} \pi\right)\right) \\
& =(-1)^{k+1} \operatorname{sign}\left(\sin \left(\frac{3 k+3}{b} \pi\right) \sin \frac{2 \pi}{3}\right)=(-1)^{k+1}
\end{aligned}
$$

Now, let us compute the sign of

$$
T_{c}(t)-T_{c}(s)=-2 \sin \left(c \frac{\tau+\sigma}{2}\right) \sin \left(c \frac{\tau-\sigma}{2}\right)=-2 \sin \left(\frac{c}{6 b}\left(m+m^{\prime}\right) \pi\right) \sin \left(\frac{c}{6 b}\left(m-m^{\prime}\right) \pi\right)
$$

We have, with $c=b+1=3 n+2$,

$$
\begin{aligned}
& \text { for } \begin{aligned}
A_{k}: \quad z(t)-z(s) & =-2 \sin c \frac{\pi}{3} \sin \left(c \frac{m-b}{3 b} \pi\right) \\
& =-2(-1)^{n} \sin \frac{2 \pi}{3} \sin \left(c \frac{n-k}{b} \pi\right) \\
& =2(-1)^{n} \sin \frac{2 \pi}{3}(-1)^{n-k} \sin \left(\frac{n-k}{b} \pi\right) \\
\text { so } \operatorname{sign}(z(t)-z(s)) & =(-1)^{k} .
\end{aligned}
\end{aligned}
$$




$$
\begin{aligned}
& \text { for } B_{k}: \quad z(t)-z(s)=-2 \sin \left(c \frac{b+3 k+2}{3 b} \pi\right) \sin \left(c \frac{\pi}{3}\right) \\
& =-2 \sin \left(\left(n+k+1+\frac{n+k+1}{b}\right) \pi\right)(-1)^{n} \sin \frac{2 \pi}{3} \\
& =2(-1)^{k} \sin \left(\frac{n+k+1}{b} \pi\right) \sin \frac{2 \pi}{3} . \\
& \text { so } \operatorname{sign}(z(t)-z(s))=(-1)^{k} \text {. } \\
& \text { for } C_{k}: z(t)-z(s)=-2 \sin \left(c \frac{2 \pi}{3}\right) \sin \left(c \frac{k+1}{b} \pi\right) \\
& \begin{aligned}
& =-2 \sin \left(\frac{4 \pi}{3}\right) \sin \left((b+1) \frac{k+1}{b} \pi\right) \\
& =-2 \sin \left(\frac{4 \pi}{3}\right)(-1)^{k+1} \sin \left(\frac{k+1}{b} \pi\right) \\
\operatorname{sign}(z(t)-z(s))= & (-1)^{k+1}
\end{aligned} \\
& \begin{aligned}
& =-2 \sin \left(\frac{4 \pi}{3}\right) \sin \left((b+1) \frac{k+1}{b} \pi\right) \\
& =-2 \sin \left(\frac{4 \pi}{3}\right)(-1)^{k+1} \sin \left(\frac{k+1}{b} \pi\right) \\
\operatorname{sign}(z(t)-z(s))= & (-1)^{k+1}
\end{aligned} \\
& \begin{aligned}
& =-2 \sin \left(\frac{4 \pi}{3}\right) \sin \left((b+1) \frac{k+1}{b} \pi\right) \\
& =-2 \sin \left(\frac{4 \pi}{3}\right)(-1)^{k+1} \sin \left(\frac{k+1}{b} \pi\right) \\
\text { so } \operatorname{sign}(z(t)-z(s))= & (-1)^{k+1} \text {. }
\end{aligned}
\end{aligned}
$$

Collecting these results we finally get

$$
\operatorname{sign}\left(D\left(A_{k}\right)\right)=1, \quad \operatorname{sign}\left(D\left(B_{k}\right)\right)=-1, \quad \operatorname{sign}\left(D\left(C_{k}\right)\right)=1 .
$$

The Conway sequence of signs is then

$$
s\left(C_{n-1}\right), s\left(B_{n-1}\right), s\left(A_{n-1}\right), \ldots, s\left(A_{0}\right)
$$

with $s\left(C_{k}\right), s\left(B_{k}\right), s\left(A_{k}\right)=(-1)^{n-1-k}$. Consequently the Conway normal form of our knot is $C\left(1,1,1,-1,-1,-1, \ldots,(-1)^{n-1},(-1)^{n-1},(-1)^{n-1}\right)$. Its Schubert fraction is $\frac{2 n+1}{2 n} \sim$ $-(2 n+1)$ and our knot is the torus knot $\mathrm{T}(2,2 n+1)$.

Remark: Note that $\mathrm{H}(3,3 n+1,3(3 n+1)-1)$ is the mirror image of $\mathrm{H}(3,3 n+1,3 n+2)$. See Proposition 8.

Remark: In [12], we obtained the torus knot $\mathrm{T}(2,2 n+1)$ as an alternate polynomial knot where $x(t)=T_{3}(t), y(t)=P(t), z(t)=Q(t)$ are polynomials and $\operatorname{deg} P=3 n+1$, $\operatorname{deg} Q=3 n+2$, that is to say the same degrees.

Because of their definitions, the symmetries of the harmonic knots are easy to find. They are either strongly negative amphicheiral if $a, b, c$ are odd, or strongly reversible. So that not every knot is an harmonic knot. We can also remark that harmonic knots are billiard knots in a convex (compact) billiard (in fact a truncated cube) 9].

On the other hand, it is not difficult to see that if we change the nature of one crossing point in the diagram of the $10_{116}$ knot, we can obtain the $8_{17}$ knot. The knot $8_{17}$ is famous because it is the first non reversible knot.

In the next paragraph, we shall see that it is possible to choose the nature of the crossing points with a (shifted) Chebyshev polynomial as height function. 


\section{Every knot is a Chebyshev knot}

Let us denote $B_{n}$ the group of $n$-braids and $S_{n}$ the symmetric group. The group of pure braids $P_{n}$ is the kernel of the morphism $\pi: B_{n} \rightarrow S_{n}$. If $\alpha$ is a braid, we shall denote $\rho(\alpha)$ its plane projection. In the next theorem, which is analogous to a theorem of Lamm for Lissajous curves (see [3, 17]), we show that

Theorem 3 Every knot has a projection which is a Chebyshev plane curve.

This is a consequence of the following proposition.

Proposition 9 Let $K$ be a knot, $b r(K)$ its bridge number. Let $m \geq \operatorname{br}(K)$ be an integer. Then $K$ has a projection which is a Chebyshev curve $x=T_{a}(t), y=T_{b}(t)$, where $a=2 m-1$, and $b \equiv 2(\bmod 2 a)$.

Proof. Let $K$ be a knot. Let $D$ be a regular diagram of $K$ such that the abscissa has only two extremal values reached at $m$ maxima and $m$ minima. It means that $K$ is the plat closure of a horizontal braid $t$ with $2 m$ strings. We can suppose the last string unbraided. Furthermore, reordering if necessary the ordinates of the $2 m$ extrema, we can suppose that $\pi(t)=(2,3) \cdots(2 m-2,2 m-1)=\pi\left(\sigma_{2} \sigma_{4} \cdots \sigma_{2 m-2}\right)$. Let us denote $s_{\text {even }}=\rho\left(\sigma_{2} \cdots \sigma_{2 m-2}\right)$, and $s_{\text {odd }}=\rho\left(\sigma_{1} \cdots \sigma_{2 m-3}\right)$.

As $\pi(t)=\pi\left(\sigma_{2} \cdots \sigma_{2 m-2}\right)$ we see that there exists $l \in \operatorname{ker}(\pi)=P_{2 m-1}$, the group of pure braids, such that $t=l \cdot \sigma_{2} \cdots \sigma_{2 m-2}$. As the braids $A_{i j}=x^{-1} \sigma_{i}^{2} x$, where $x=\sigma_{i+1} \cdots \sigma_{j}$, generate $P_{2 m-1}$, the braid $l$ is a composition of such elementary braids. It is not difficult to see that there is a braid $\alpha_{i j}$ equivalent to $A_{i j}$ with plane projection $\rho\left(\alpha_{i j}\right)=\left(s_{\text {even }} s_{\text {odd }}\right)^{a}$. Consequently, the braid $t$ is equivalent to a braid projecting on $\left(s_{\text {even }} s_{\text {odd }}\right)^{k a} s_{\text {even }}$. Using the braid description of Chebyshev curves (corollary 1), we conclude that our knot $K$ is equivalent to a knot projecting upon the Chebyshev curve $x=T_{a}(t), y=T_{b}(t)$, $a$ odd, $b \equiv$ $2(\bmod 2 a)$.

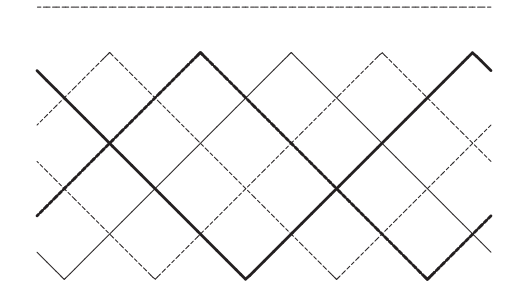

Figure 12: The "plane braid" ( $\left.s_{\text {even }} s_{\text {odd }}\right)^{5}$ is the projection of the braid $\alpha_{2,5} \in B_{5}$.

We shall prove our principal result with a density argument based on Kronecker's theorem ([7], Theorem 443, p. 382.) Let us recall this theorem. 
Theorem 4 (Kronecker) If $\theta_{1}, \theta_{2}, \ldots, \theta_{k}, 1$ are linearly independent over $\mathbf{Q}$, then the set of points $\left(\left(n \theta_{1}\right),\left(n \theta_{2}\right), \ldots,\left(n \theta_{k}\right)\right)$ is dense in the unit cube. Here $(x)$ denotes the fractional part of $x$.

We shall need the following lemma.

Lemma 2 Let $c_{1}, \ldots, c_{k}$ be real numbers such that $-1<c_{1}<c_{2}<\cdots<c_{k}<1$. There exists a positive number $e<1-c_{k}$ such that the numbers $\arccos \left(c_{1}+e\right)$, $\arccos \left(c_{2}+\right.$ $e), \ldots, \arccos \left(c_{k}+e\right), 1$ are linearly independent over $\mathbf{Q}$.

Proof. First, we shall prove, by induction on $k$, that the functions $\arccos \left(c_{i}+x\right), 1$ are linearly independent over $\mathbf{R}$. Let $\lambda_{0}+\sum_{i=1}^{k} \lambda_{i} \arccos \left(c_{i}+x\right)=0$ be a linear relation between these functions. We get by derivating

$$
\sum_{i=1}^{k} \lambda_{i} \frac{1}{\sqrt{1-\left(c_{i}+x\right)^{2}}}=0 .
$$

Then, when $x \rightarrow 1-c_{k}$, we get $\lambda_{k}=0$, and the result follows by induction.

Suppose now that for each $e<1-c_{k}$ there exists a relation

$$
\lambda_{0}+\sum_{i=1}^{k} \lambda_{i} \arccos \left(c_{i}+e\right)=0, \quad \lambda_{i} \in \mathbf{Q}, \quad \sum_{i=1}^{k}\left|\lambda_{i}\right|=1 .
$$

By cardinality, there are infinitely many $e$ in $\left(0,1-c_{k}\right)$ with the same collection of $\lambda_{i}$. This means that the analytic function $\lambda(x)=\lambda_{0}+\sum_{i=1}^{k} \lambda_{i} \arccos \left(c_{i}+x\right)$ has an infinity of zeroes in the interval $\left(0,1-c_{k}\right)$, which is absurd.

Theorem 5 Every knot is a Chebyshev knot.

Proof. Let $K$ be a knot projecting on the Chebyshev curve $\left\{x=T_{a}(t), y=T_{b}(t)\right\}$. The crossing points of the projection are obtained for the distinct pairs of values

$$
t=\cos \left(\frac{k}{a}+\frac{h}{b}\right) \pi, s=\cos \left(\frac{k}{a}-\frac{h}{b}\right) \pi, \frac{k}{a}+\frac{h}{b}<1 .
$$

Let us denote these values by $\left(t_{i}, s_{i}\right), i=1 \ldots n=\frac{1}{2}(a-1)(b-1)$. By our lemma, let $e<1-\cos \frac{\pi}{a b}$ be a positive number such that the $2 n+1$ numbers $1, \tau_{i}=\arccos \left(t_{i}+e\right), \sigma_{i}=$ $\arccos \left(s_{i}+e\right), i=1, \ldots n$, are linearly independent over $\mathbf{Q}$. Let us define the function $Z(t)=T_{c}(t+e)$ (depending on the integer $c$ ). We have

$$
Z\left(t_{i}\right)-Z\left(s_{i}\right)=\cos c \tau_{i}-\cos c \sigma_{i}
$$

Since the numbers $1, \tau_{i}, \sigma_{i}$ are linearly independent over $\mathbf{Q}$, the numbers $c \tau_{i}(\bmod 2 \pi)$ and $c \sigma_{i}(\bmod 2 \pi)$ are dense in $[0,2 \pi]^{2 n}$ by Kronecker's theorem. So that we can choose arbitrarily the signs of $Z\left(t_{i}\right)-Z\left(s_{i}\right)$, that is, the over/under nature of the crossing points. 


\section{Example 1: the knot $6_{1}$}

Let us consider the curve parametrized by

$$
x=T_{3}(t), y=T_{8}(t), z=T_{10}\left(t+\frac{1}{100}\right) .
$$

Computing sign $(D)$ for the $\frac{1}{2}(3-1)(8-1)=7$ crossing points, we find the Conway normal

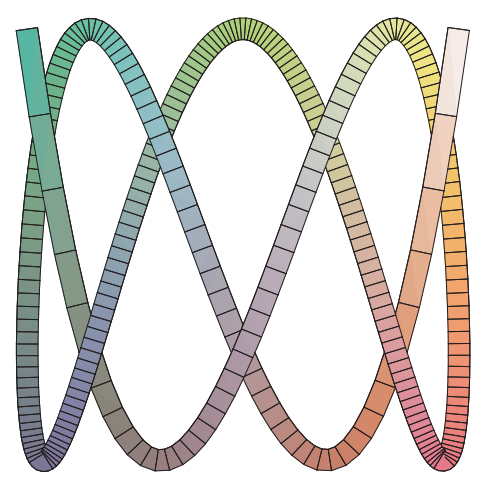

Figure 13: The knot $6_{1}$ is a Chebyshev knot

form: $[-1,-1,-1,-1,1,1,1]$. Its Schubert fraction (see [18]) is then

$$
-1+\frac{1}{-1+\frac{1}{-1+\frac{1}{-1+\frac{1}{1+\frac{1}{1+\frac{1}{1}}}}}}=\frac{9}{-5} \sim \frac{9}{4} .
$$

This knot is the knot 61 .

\section{Example 2: the knot $8_{17}$}

The famous $8_{17}$ knot is non reversible and strongly (-)amphicheiral (see [10] p. 128). It is a 3-bridge knot. The Chebyshev curve $T_{6}(x)=T_{5}(y)$ is one of its diagrams. It can be parametrized by $x=T_{5}(t), y=T_{6}(t), z=T_{33}\left(t+148 \cdot 10^{-4}\right)$. Its reverse can be parametrized by $x=T_{5}(t), y=T_{6}(t), z=T_{33}\left(t-148 \cdot 10^{-4}\right)$. We see that it is the reversed image of $8_{17}$ by a half-turn about the $y$ axis.

\section{Conclusion}

Let us give a list of the first 2-bridge harmonic knots with their Conway-Rolfsen numbering. Because of their simplicity, we also give their Chebyshev diagrams. A bar over a knot name 


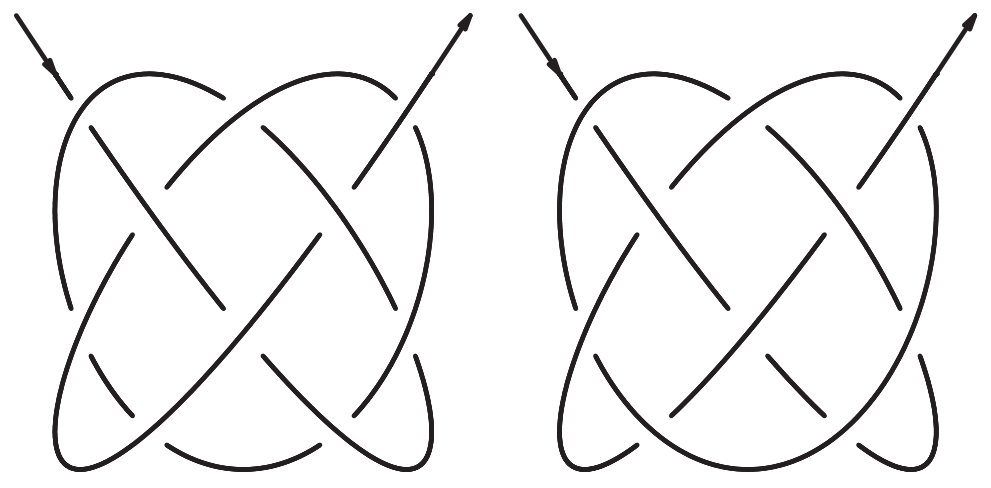

Figure 14: The knot $8_{17}$ and its reverse as Chebyshev knots

indicates mirror image.

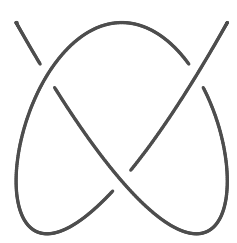

$\overline{3}_{1}$

$\mathrm{H}(3,4,5)$

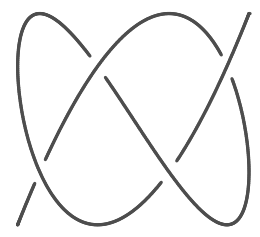

41

$\mathrm{H}(3,5,7)$

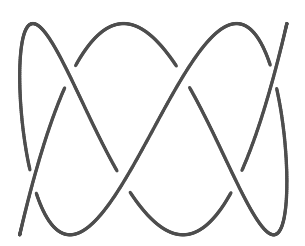

$\overline{5}_{1}$

$\mathrm{H}(3,7,8)$

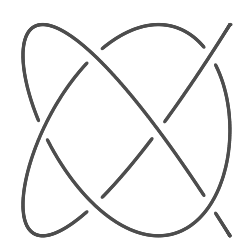

$\overline{5}_{2}$

$\mathrm{H}(4,5,7)$

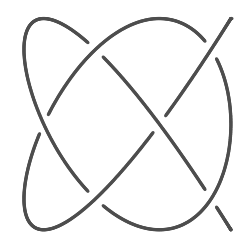

62

$\mathrm{H}(4,5,11)$

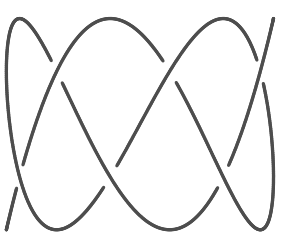

63

$\mathrm{H}(3,7,11)$

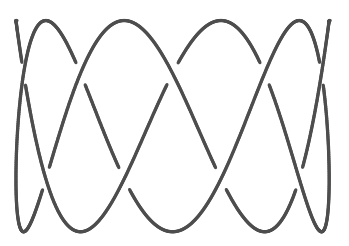

$\overline{7}_{1}$

$\mathrm{H}(3,10,11)$

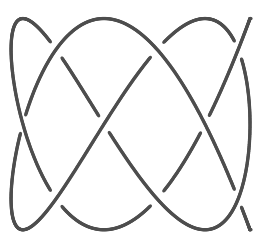

$7_{5}$

$\mathrm{H}(4,7,9)$

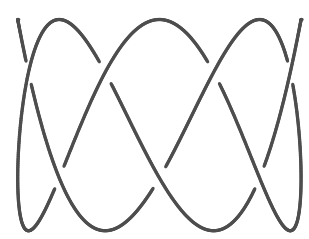

$\overline{7}_{7}$

$\mathrm{H}(3,8,13)$

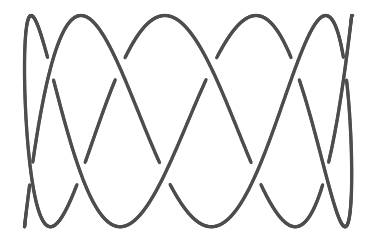

83

$\mathrm{H}(3,11,13)$

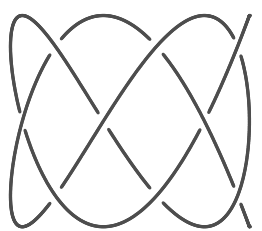

$8_{7}$

$\mathrm{H}(4,7,13)$

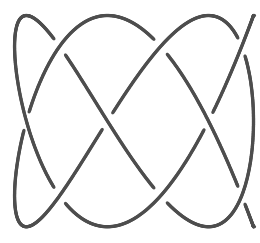

$9_{20}$

$\mathrm{H}(4,7,17)$ 


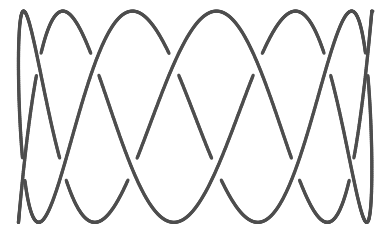

$\overline{9}_{1}$

$\mathrm{H}(3,13,14)$

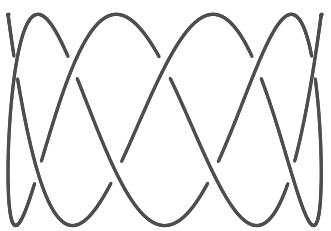

$\overline{9}_{31}$

$\mathrm{H}(3,10,17)$

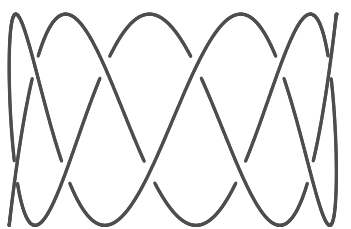

$\overline{9}_{17}$

$\mathrm{H}(3,11,16)$

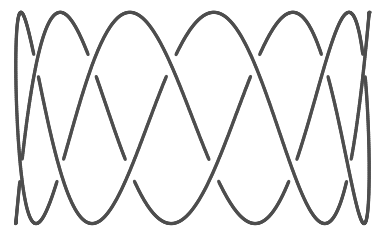

$10_{37}$

$\mathrm{H}(3,13,17)$

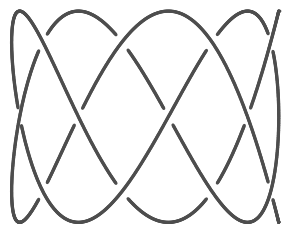

$\overline{9}_{18}$

$\mathrm{H}(4,9,11)$

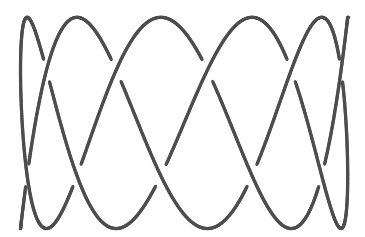

$10_{45}$

$\mathrm{H}(3,11,19)$

In [15], we give a complete list of Chebyshev parametrizations of the 2-bridge knots of 10 crossings or less.

Now, let us give the list of harmonic knots $\mathrm{H}(5,6, c)$. We get $4=\frac{1}{2} \varphi(5) \varphi(6)$ different types up to mirror symmetry.

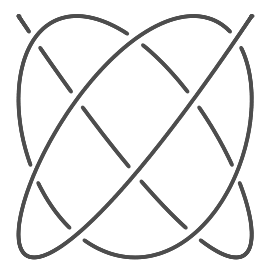

0

$\mathrm{H}(5,6,1)$

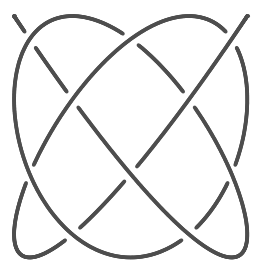

52

$\mathrm{H}(5,6,7)$

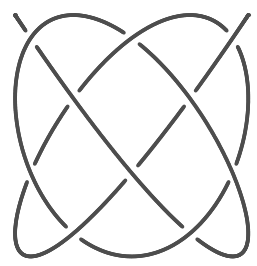

$10_{159}$

$\mathrm{H}(5,6,13)$

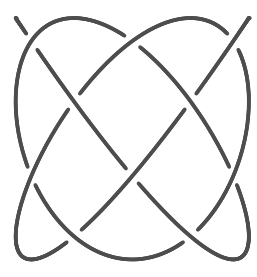

$\overline{10}_{116}$

The $10_{159}$ knot is the harmonic knot $\mathrm{H}(5,6,13)$. Its bridge number is equal to 3 . Our Chebyshev parametrization provides an easy proof that it is strongly reversible (compare [10], Appendix F, p. 254).

In conclusion, we have found a great number of distinct harmonic knots. Furthermore, their diagrams have a small number of crossing points. We hope that our Chebyshev models will be useful for the study of knots.

In [13], we classify the harmonic knots $\mathrm{H}(3, b, c)$ and $\mathrm{H}(4, b, c)$. Even for $a=5$, the classification of harmonic knots seems to be a difficult and interesting problem.

\section{References}

[1] J. S. Birman, Braids, Links and Mapping Class Groups, Ann. of Math. Studies 82, Princeton University Press, 1974.

[2] M. G. V. Bogle, J. E. Hearst, V. F. R. Jones, L. Stoilov, Lissajous knots, Journal of Knot Theory and its Ramifications, 3(2), (1994), 121-140. 
[3] A. Boocher, J. Daigle, J. Hoste, W. Zheng, Sampling Lissajous and Fourier knots, Experiment. Math., Vol 18(4), (2009), 481-497.

[4] E. H. Comstock, The Real Singularities of Harmonic Curves of three Frequencies, Trans. of the Wisconsin Academy of Sciences, Vol XI, (1897) 452-464.

[5] G. Fischer, Plane Algebraic Curves, A.M.S. Student Mathematical Library Vol. 15, 2001.

[6] G. Freudenburg, J. Freudenburg, Curves defined by Chebyshev polynomials, 19 p., (2009), arXiv:0902.3440

[7] G.H. Hardy, E.M. Wright, An Introduction to the Theory of Numbers, 4th edition, Oxford University Press, 1960.

[8] J. Hoste, L. Zirbel, Lissajous knots and knots with Lissajous projections, Kobe J. Math. 24(2) (2007), 87-106.

[9] V. F. R. Jones, J. Przytycki, Lissajous knots and billiard knots, Banach Center Publications, 42, (1998), 145-163.

[10] A. Kawauchi, editor, A Survey of Knot Theory, Birhäuser, 1996.

[11] P. - V. Koseleff, D. Pecker, On polynomial torus knots, Journal of Knot Theory and its Ramifications, Vol. 17(12), (2008), 1525-1537.

[12] P. -V. Koseleff, D. Pecker, A polynomial parametrization of torus knots, Journal of Applicable Algebra in Engineering, Communication and Computing, Vo. 20 (5-6), (2009), 361-377.

[13] P. -V. Koseleff, D. Pecker, Chebyshev diagrams for rational knots, 39p., 34 figures, (2008), arXiv:0906.4083.

[14] P. -V. Koseleff, D. Pecker, On Fibonacci knots, to appear in Fibonacci Quarterly, 7p. (2009), arXiv:0908.0153.

[15] P. -V. Koseleff, D. Pecker, F. Rouillier, The first rational Chebyshev knots, Mega Conference Barcelona, 22p., 27 figures, 3 tables, (2009), submitted, arXiv:0911.0566.

[16] C. Lamm, There are infinitely many Lissajous knots, Manuscripta Math. 93, (1997), 29-37.

[17] C. Lamm, Cylinder knots and symmetric unions (Zylinder-knoten und symmetrische Vereinigungen), Ph.D. Thesis, Bonner Mathematische Schriften 321, Bonn, 1999.

[18] K. Murasugi, Knot Theory and its Applications, Boston, Birkhäuser, 341p., 1996. 
[19] D. Pecker, Simple constructions of algebraic curves with nodes, Compositio Math. 87, (1993), 1-4.

[20] J. H. Przytycki, Symmetric knots and billiard knots, Chapter 20 of the book Ideal Knots, Vol. 19 in Series on Knots and Everything, Ed. A.Stasiak, V.Katrich, L.Kauffman, World Scientific, 1999, 374-414.

[21] A. Ranjan and R. Shukla, On polynomial representation of torus knots, Journal of Knot Theory and its Ramifications, Vol. 5, (2), (1996), 279-294.

[22] D. Rolfsen, Knots and Links, Math. Lecture Series 7, Publish or Perish (1976).

[23] A. R. Shastri, Polynomial representation of knots, Tôhoku Math. J. 44, (1992), $11-17$.

[24] J. C. Turner, On a class of knots with Fibonacci invariant numbers, Fibonacci Quarterly 24, 1, (1986), 61-66.

[25] V. A. Vassiliev, Cohomology of knot spaces, Theory of Singularities and its Applications, Advances Soviet Maths Vol. 1, (1990) 